\title{
The Study of Instructional Explanations in Classroom Teaching of the Graduate Course "Pattern Recognition"
}

\author{
Huang Ling, Shi Yuqiu, and Hu Bo \\ College of Electric and Information Engineering, Guangxi University of Science and Technology, Liuzhou, Guangxi, China \\ (huangling79hl@163.com)
}

\begin{abstract}
Instructional explanations are the important part in classroom teaching. It is also a key step to improving the teaching effective of the graduate course "pattern recognition". The purpose of instructional explanations in classroom teaching is first introduced according to the teaching purpose of graduate course "pattern recognition". Then the instructional explanations of different classroom teaching content are discussed. And several noticeable problems of the instructional explanations in the classroom teaching of the graduate course "pattern recognition" are proposed. It should be helpful for improving the effective of the instructional explanations in classroom teaching of the graduate course "pattern recognition".
\end{abstract}

Keywords - Instructional explanations, classroom teaching, pattern recognition

\section{研究生课程《模式识别》课堂教学中教学解释的研究}

\author{
黄玲 石玉秋 胡波 \\ 广西科技大学电气与信息工程学院，柳州，广西，中国
}

\begin{abstract}
摘 要 教学解释是课堂教学的重要组成部分, 这也是改善研究生课程《模式识别》教学效果的关键步骤。首先根据研究生课程 《模式识别》的教学目的介绍了教学解释在课堂教学中的作用, 然后对不同课堂教学内容中的教学解释进行了讨论, 最后提出了在研 究生课程《模式识别》课堂教学中教学解释值得注意的几个问题。这将有助于改善研究生课程《模式识别》课堂教学中教学解释的教 学效果。
\end{abstract}

关键词 教学解释, 课堂教学, 模式识别

\section{1. 引言}

教学解释是指教师在课堂教学中对于知识点进行的解 释, 教学解释是课堂教学的核心教学方式, 所以教学解释 一直是教学改革研究的重点 ${ }^{[1][2][3]}$ 。

《模式识别》是控制科学与工程学科的重要专业课。 课程涉及的内容范围广, 具有较完整的理论体系和较强的 应用背景。所以, 在课堂教学中, 为了提高课堂教学效果, 对于课堂教学中教学解释的教学改革就成为了关键。

从学习心理学的角度出发看, 教师在课堂上的教学解 释主要目的是为了让学生对相关知识点形成自我解释, 完 成对知识点的理解。所以, 为提高《模式识别》教学解释 的效果应该围绕学生自我解释进行相关的教学改革。

广西科技大学 2013 年度研究生课程建设项目支持

\section{2. 《模式识别》课堂教学中教学解释的作用}

在对《模式识别》课堂教学中教学解释进行教学改革 前, 首先需要明确教学解释在课堂教学中的作用。一般认 为教学解释的作用主要是为了完成学生的自我解释。在《模 式识别》的研究生课程中, 学生的自我解释, 主要是对相 关概念、理论及其应用的一种自我解释。因此, 可以考虑 从《模式识别》的教学目的进行教学解释作用的分析。

《模式识别》是一门既有完整理论体系又具有较强实 践要求的研究生专业课。其完整的理论体系使得 “模式识 别” 一直被认为是 “控制科学与工程” 一级学科下的一个 二级学科, 同时其广泛的应用使得在许多研究生专业将其 作为专业课开设。所以, 针对这一现象, 《模式识别》的教 学重点在于学生能力的培养。无论理论教学还是上机实践 都是为了培养学生应用模式识别的知识和技能在相关领域 
进行模式识别工作的能力。因此, 对研究生进行模式识别 相关工作能力的培养是课程的教学目的。

从学习心理学角度看, 在《模式识别》课堂中教学解 释是为了促进学生的自我解释。结合课程的教学目的, 这 主要体现在三个方面: 一是学生完成对相关概念和理论的 自我解释; 二是将这些概念和理论与学生自己已有的知识 体系融为一体, 完成对相关概念和理论的理解; 三是形成 如何使用模式识别知识和理论进行工作的一个流程和认 识, 完成一个对应用模式识别如何进行工作的自我解释。 所以, 在《模式识别》的课堂教学中教学解释就是促进学 生在这三个方面形成较为正确合理的自我解释。

\section{3. 不同课堂教学内容中的教学解释}

在《模式识别》的课堂教学中, 根据要求学生完成自 我解释的差异可以将教学内容分成三类: 一是《模式识别》 的概念与理论; 二是模式识别理论的应用范围和推导过程; 三是对相关问题进行模式识别的方法。针对不同的教学内 容, 教学解释也相应的存在差异。

\section{1 概念理论}

《模式识别》作为一门研究生专业课, 具有较为完备 的理论体系。在教学中, 一般要求《矩阵论》《数理统计》 等相关数学课程为先修课程, 要求学生具备一定的数学基 础。因此, 在教学实践中, 对于概念理论的讲解往往注重 其数学基础。但是, 这样的实际教学效果并不好。学生对 于相关概念和理论的理解相对较为死板, 对后续教学的帮 助不大。可以认为, 这样的教学解释并没有促进学生的自 我解释, 学生对于相关教学内容的自我解释是孤立的知识 点, 并没有从心理上完成了对模式识别概念和理论的解释 工作。所以, 在这一类教学解释中, 要注重《模式识别》 课程数学基础的同时结合工程实践的例子, 促进学生对相 关概念和理论的理解。

比如在 Bayes 理论的教学中, Bayes 理论需要不少统计 学、概率论的基础知识, 这使得单纯的数学推导教学解释 让许多研究生无法很好的完成自我解释。实际教学中, 即 使在相关推导证明的题目中取得较高分数的学生也常常在 实际应用中也无法联想到相关知识。而在教学改革实践中, 通过加入一个工程实践的举例, 教学效果得到极大改善。 学生在后续模式识别应用实例的学习中, 碰到使用 Bayes 理论的情况就会自动的结合所举例的工程实例进行类比, 从而形成学习迁移, 促进后续知识的学习。这就表明学生 对于 Bayes 理论的自我解释取得了较好的效果, 相关的教 学解释也是较为成功的。

\section{2 理论的推导与应用范围}

模式识别相关理论的推导是确定模式识别方法理论应 用范围的基础, 根据不同的教学内容, 推导的过程存在一 定的差异。而在实际工程实践中模式识别理论的推导过程 一般都是容易被学生忘记的教学内容。因此, 在这一内容 的教学中, 真正的教学目标是希望学生完成模式识别理论 与自己已有专业知识体系的融合, 从自己已有的专业知识 体系去对模式识别理论进行自我解释。这一过程的完成因 为学生的个人差异而不同。

由于这一阶段学生自我解释的重大差异, 教师给出的 教学解释主要引导学生去完成自我解释。这些教学解释应 该更注重方向性, 而不应重视具体的内容。具体的解释形 式可以采取提问、讨论的形式, 不强求统一的形式, 只要 求学生接纳模式识别相关理论。

\section{3 具体应用问题分析与程序讲解}

模式识别是一门实践性很强的课程, 在课堂教学中就 体现在具体工程问题的分析以及相关程序的讲解中。虽然 在工程实践中模式识别的方法很多, 但是在学生第一次接 触相关理论的应用时, 应该明确的给出学生一个范例。这 一阶段就是让学生完成一个如何应用模式识别理论进行工 程实践的自我解释。所以, 相应的教学解释要求明确而直 接, 就是让学生形成一个模板式的印象。

\section{4. 《模式识别》课堂教学中教学解释值得注意的问题}

教学解释与自我解释是解释理论中两个相互依存的概 念, 在课堂教学中可以进行改革的教师的教学解释, 但是 真正起作用的是学生的自我解释, 因此教学解释是以促进 学生自我解释为目的的。而在《模式识别》的课堂教学中, 根据课程的特点, 以下几个问题值得注意。

\section{1 学生差异的对教学解释的影响}

由于研究生扩招及跨专业考研等客观现象的存在, 在 教学实践中经常出现学生个体间存在巨大差异的情况。这 使得学生已有的知识体系存在巨大差异, 因此同样的教学 解释对于不同学生的自我解释会有不同的效果, 对此要考 虑多层次、多角度对问题进行教学解释。

\section{2 辅助教学手段的应用}

随着计算机技术的不断发展, 多媒体技术的应用使得 很多教学内容可以多样化的形式展现在学生面前。在教学 解释中引入各种模型、视频等辅助教学手段是提高教学解 释质量的有效途径。 


\section{5. 小结}

针对研究生课程《模式识别》的课堂教学, 提出以促 进学生自我解释为目的从教学解释出发进行教学改革, 这 将为《模式识别》教学质量的提高提供理论基础。

\section{参考文献(References)}

[1] Björn B. de Koning, Huib K. Tabbers, Remy M.J.P. Rikers and Fred Paas. Learning by generating vs. receiving instructional explanations: Two approaches to enhance attention cueing in animations. Computers \& Education, Volume 55, Issue 2, pp. 681-691,2010

[2] Emilio Sánchez and Héctor García-Rodicio. Using online measures to determine how learners process instructional explanations. Learning and Instruction, Volume 26, pp. 1-11 2013

[3] J. Elizabeth Richey and Timothy J. Nokes-Malach, How much is too much? Learning and motivation effects of adding instructional explanations to worked examples. Learning and Instruction, Volume 25, pp. 104-124,2013 